\title{
179. On the Diffraction Enhancement of Symmetry in SiC
}

\author{
By Ryoichi Sadanaga,*) Kazumasa OHsumI,*) \\ and Takeo MATsumoto**)
}

(Comm. by Tei-ichi Iтo, M. J. A., Dec. 12, 1973)

Its cubic modification $3 C$ set aside, the polytype of $\mathrm{SiC}$ is either hexagonal $\left(P 6_{3} m c\right)$ or trigonal $(P 3 m$ or $R 3 m)$ in symmetry, the corresponding Laue symmetry being $6 / \mathrm{mmm}$ or $\overline{3} \mathrm{~m}$. However, polytypes of the latter symmetry sometimes produce an X-ray pattern of $6 / \mathrm{mmm}$. This seems to be a typical example of diffraction enhancement of symmetry (Sadanaga and Takeda, 1968).

Ramsdell and Kohn (1951) have shown that the diffraction pattern of a trigonal polytype of $\mathrm{SiC}$ is hexagonal if its structure conforms to the condition that it is made up of two parts related to each other reflection-symmetrically so as, using the Zhdanov notation, $2332 \Rightarrow A B C B A C A B C B \Rightarrow 10 H$ (Fig. 1), and they each consist of more than one number (when they consist of only one number each, the structure itself is hexagonal, namely, $22 \Rightarrow A B C B \Rightarrow 4 H, 33 \Rightarrow A B C B A C$ $\Rightarrow 6 H$ and so forth). Let us explaining the diffraction enhancement of symmetry of SiC satisfying this condition, say the Ramsdell condition***), on the basis of the vector set theory (Ohsumi et al., 1972; Sadanaga et al., 1973).

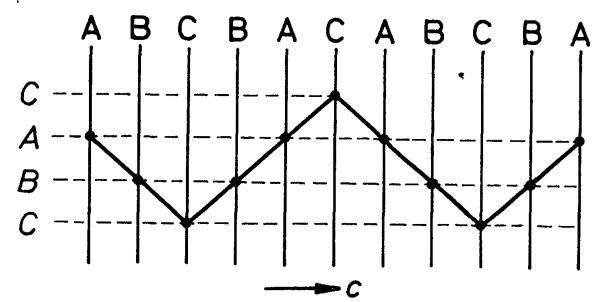

Fig. 1. Layer-sequence in SiC polytype 10H, 2332. Horizontal broken lines indicate the position of the origin for each layer.

The unit layer of $\mathrm{SiC}$ is built up of $\mathrm{Si}-\mathrm{C}$ pairs, $\mathrm{Si}$ and $\mathrm{C}$ being linked together like a dumbbell with unequal weights and with the axis parallel to the $c$ axis of the crystal. Then, if the Friedel law

*) Mineralogical Institute, Faculty of Science, University of Tokyo.

**) Department of Earth Sciences, Faculty of Science, Kanazawa University.

***) SiC polytypes satisfying the Ramsdell condition are not always trigonal; they can be hexagonal as in the case of 323323 . 


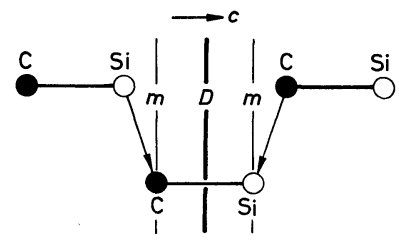

Fig. 2. Si-C dumbbells in symmetrically arranged layers. $D$ indicates a dichroic plane relating C to $\mathrm{Si}$ and vice versa, and two $\mathrm{m}$ 's are ordinary reflection planes, one for $\mathrm{C}$ and the other for $\mathrm{Si}$.

holds, the vector from $\mathrm{Si}$ to $\mathrm{C}$ in the left half of Fig. 2 is associated with the same weight as that for the vector from $\mathrm{C}$ to $\mathrm{Si}$ in the right half of the figure; this pair of vectors are symmetric up to both position and weight with respect to the plane $D$ perpendicular to the $c$ axis. The same situation holds between every Si-C or C-Si vector and its counterpart with respect to plane $D$, Si-Si vectors are reflection-symmetrically distributed in pairs, and the same applies to $\mathrm{C}-\mathrm{C}$ vectors. The vector set of this structure is thus reflectionsymmetric with respect to a plane perpendicular to the $c^{*}$ axis and through the origin of reciprocal space. Now, if we add the reflection to $\overline{3} \mathrm{~m}$, we obtain $6 / \mathrm{mmm}$; the symmetry of the X-ray diffraction pattern of the crystal should be not trigonal but hexagonal. The plane $D$ is looked upon as a black-and-white plane (dichroic plane) which is the product of an ordinary reflection operation and a colourchanging operation and relates a pair of different kinds of atom situated symmetrically. Each unit layer of $\mathrm{SiC}$ has a dichroic plane in the middle and parallel to the layer. Therefore, the Ramsdell condition is equivalent to saying that the entire structure is related to its mirror image by a dichroic plane.

The next question is whether there is a structure of SiC, in which the Ramsdell condition is not satisfied and yet diffraction enhancement takes place. Since a general procedure to answer this question is lacking and to examine the symmetry of the vector set for all conceivable polytypes of $\mathrm{SiC}$ is practically impossible, we only demonstrate here the possibility of deriving such a structure from a stack of two kinds of layer.

Suppose that the structure of a polytype can be looked upon as a stack of two kinds of layer $X$ and $Y$, each being composite of $A, B$ and $C$, and that each of $X$ and $Y$ is symmetric with respect to the $A B C$ sequence. This symmetry creates a dichroic plane in the middle of every $X$ and $Y$ (Fig. 3(a)). Then, according to the theorem that in an arbitrary stack of two kinds of layer $X$ and $Y$, the number of $X-Y$ couples is equal to the number of $Y-X$ couples if the distance of 


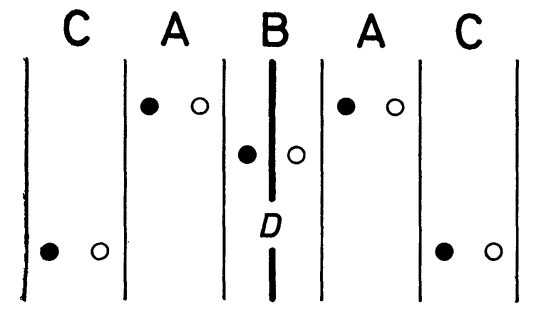

(a)

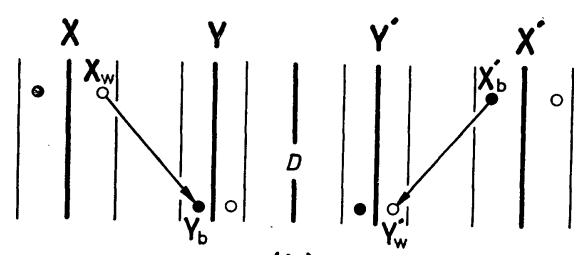

(b)
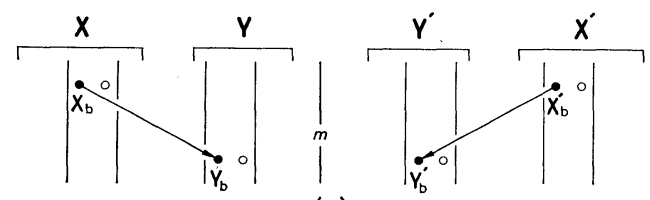

(c)

Fig. 3. Symmetries in the distribution of interatomic vectors.

(a) Symmetrically arranged unit layers producing a dichroic plane in the middle.

(b) Interatomic vectors between different kinds of atom in a stack of two kinds of layer, each bearing a dichroic plane in the middle. Note that $X_{w} \rightarrow Y_{b}$ is related to $X_{b}^{\prime} \rightarrow Y_{w}^{\prime}$ by the dichroic plane $D$.

(c) Interatomic vectors between atoms of the same kind in a stack of two kinds of layer $X$ and $Y$, each being a symmetric composite of $A, B$ and $C$ as in (a). Note that $X_{b} \rightarrow Y_{b}$ is related to $X_{b}^{\prime} \rightarrow Y_{b}^{\prime}$ by the ordinary reflection plane $m$.

separation between $X$ and $Y$ in the former is the same as that between $Y$ and $X$ in the latter (Marumo and Saito, 1972), we can conclude that the interatomic vector between every pair of different kinds of atom, $X_{w} \rightarrow Y_{b}$ for example, is symmetrically related to its counterpart, $X_{b}^{\prime} \rightarrow Y_{w}^{\prime}$, by a dichroic operation (Fig. 3(b)). On the other hand, because of the symmetric arrangement of unit layers within $X$ and $Y$, and by dint of the above theorem, we find that the interatomic vector between every pair of atoms of the same kind, $X_{b} \rightarrow Y_{b}$ for example, is symmetrically related to its counterpart, $X_{b}^{\prime} \rightarrow Y_{b}^{\prime}$, by an ordinary reflection operation (Fig. $3(\mathrm{c})$ ). Therefore, as long as the Friedel law holds, the vector set of the structure exhibits a symmetry according to a reflection plane through the origin of reciprocal space and parallel to the layers; the X-ray diffraction pattern of the crystal is reflection-symmetric with respect to the above reflection plane even if the structure bears no such symmetry.

Next, let us take a stack of two kinds of layers according to the sequence $X Y X Y Y X$ which is the simplest among asymmetric types of sequence of two kinds of layer. In order to derive a desired model of polytype based upon this two-layer sequence, we substitute symmetric stacks of $A, B$ and $C$ for $X$ and $Y$, where no $A, B$ and $C$ are 


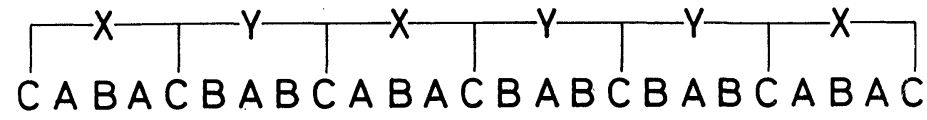

Fig. 4. Model of 24-layer SiC polytype derived from two-layer sequence $X Y X Y Y X$.

allowed to follow the same. Also, since we are concerned with SiC polytypes, we must avoid those structures whose Zhdanov notation contains 1, namely, those that comprise one of $A B A B, B C B C$ and $C A C A$ sequences, because such alternating sequences are believed to appear only in the special polytype $2 H$.

A short series of trial will then reveal that all the simple substitutions yield either hexagonal structures or those conforming to the Ramsdell condition, until we arrive at the model of a 24-layer structure, 44422422, by the substitutions, $X=\left(\frac{1}{2} C\right) A B A\left(\frac{1}{2} C\right)$ and $Y=\left(\frac{1}{2} C\right) B A B\left(\frac{1}{2} C\right)$, where $\left(\frac{1}{2} C\right)$ means the inclusion of a half of layer $C$ (Fig. 4). As evident from its Zhdanov notation, this structure is neither hexagonal nor in conformity with the Ramsdell condition, while the symmetry of its X-ray diffraction pattern is reflectionsymmetric with respect to a plane perpendicular to the $c^{*}$ axis and through the origin of reciprocal space, namely, it is hexagonal, $6 / \mathrm{mmm}$. It will then be easily recognized that other polytypes derived by more complicated substitutions, such as $X=\left(\frac{1}{2} C\right) A B C B A\left(\frac{1}{2} C\right)$ and $Y=\left(\frac{1}{2} C\right) B A B\left(\frac{1}{2} C\right)$, can also occasion diffraction enhancement of symmetry though they do not realize the Ramsdell condition.

In conclusion, we note that whenever the structure determination of a polytype of $\mathrm{SiC}$ with a long $c$ period and with the diffraction pattern hexagonal in symmetry is attempted at, the possibility of enhancement of symmetry should be borne in mind.

Acknowledgment. The authors wish to express their sincere gratitude to Professor Tei-ichi ITo, M. J. A., for his valuable suggestions, constant encouragement, and critically reviewing their manuscript.

\section{References}

Marumo, F., and Saito, Y. (1972): Acta Cryst., B28, 867.

Ohsumi, K., Okamura, F. P., and Sadanaga, R. (1972): Acta Cryst., A28, S121. Ramsdell, L. S., and Kohn, J. A. (1951) : Acta Cryst., 4, 111.

Sadanaga, R., Ohsumi, K., and Matsumoto, T. (1973) : Proc. Japan Acad., 49, 609. Sadanaga, R., and Takeda, H. (1968): Acta Cryst., B24, 144. 